\title{
The effective complex permittivity stability in filled polymer nanocomposites studied above the glass transition temperature
}

\author{
F. Elhaouzi ${ }^{1,2}$, A. Mdarhri ${ }^{1}$, M. Zaghrioui ${ }^{3,4}$, C. Honstettre ${ }^{4}$, I. El Aboudi ${ }^{1}$, A. Belfkira ${ }^{2}$ and M. El Azhari ${ }^{1}$ \\ ${ }^{1}$ FSTG -Cadi Ayyad University, LMCN, Marrakesh, Morocco \\ ${ }^{2}$ FSTG -Cadi Ayyad University, LBCM, Marrakesh, Morocco \\ ${ }^{3}$ François Rabelais University, GREMAN UMR 7347-CNRS,IUT, Blois, France \\ ${ }^{4}$ François Rabelais University, GREMAN UMR 7347-CNRS, UFR Sciences, Tours, France
}

*Corresponding authors: a.mdarhri@uca.ma and elhaouzifatiha@gmail.com

\begin{abstract}
The temperature effecton the dielectric response of nanocomposite at low frequencies range is reported. The investigated samples are formed by a semi-crystalline ethylene-co-butyl acrylate (EBA) polymer filled with three concentrations of the dispersed conducting carbon black (CB) nanoparticles. The temperature dependence of the complex permittivity has been analyzedabove the glass transition temperature of the neat polymer matrix $T_{g}=-75^{\circ} \mathrm{C}$. For all $\mathrm{CB}$ concentrations, the dielectric spectra follow a same trend in frequency range $100-106 \mathrm{~Hz}$. More interestingly, the stability of the effective complex permittivity $\varepsilon=\varepsilon^{\prime}-i \varepsilon^{\prime \prime}$ with the temperature range of $10-70^{\circ} \mathrm{C}$ is explored. While the imaginary part of the complex permittivity $\varepsilon^{\prime \prime}$ exhibits a slight decreasewith temperature, the real part $\varepsilon^{\prime}$ shows a significant reduction especially for high loading samples. The observed dielectric response may be related to the breakup of the three-dimensional structurenetwork formed by the aggregation of CB particles causing change at the interfaceEBA-CB.This interface is estimated bythe volume fraction of constrained polymer chain according to loss tangent data of dynamic mechanical analysis.
\end{abstract}

\section{Introduction}

The addition of nanoparticles to a polymer matrix has received much attention over several decades[1]. Nanoparticles offer improved mechanical, electrical and thermal properties of polymer systems [2]. Dueto their interesting dielectric properties, nanocomposite materials with polymeric matrix have a great potential for advanced applicationssuch as electromagnetic shielding materials, dielectric resonator antennas, capacitors, electro-active materials to cite a few[3,4,5]. Challenge now is focused on increasing the polymer permittivity without compromising the other characteristics especially ease and quality of polymer processing. For example high permittivity values are needed for reducing the applied electric field to actuate electro-active materials for artificial muscle applications [6]or for increasing the energy density of capacitors [7].

Carbon Black (CB) nanoparticles filled elastomers are among the most commonly nanocomposite widely studied because lightweight, large area, low cost, high flexibility [8], and temperature requirements exist. In addition, the use of nanoscale high dielectric constant fillers make possible to manufacturing polymer thin films at thickness of the order of microns. This study aims at the understanding the temperature dependence of theeffective complex permittivity $\varepsilon=\varepsilon^{\prime}-i \varepsilon^{\prime \prime}$ of ethylene-co-butyl acrylatecopolymer (EBA) filled with spherical nanoparticles of CB. Three composite samples containing with fraction volume of $\mathrm{CB}$ varying from 6.8 to $19.9 \%$ have been examined. Thedirect current electrical conductivity $\sigma_{D C}$ versus temperature of this series of composites have been already reported by us elsewhere [9].From that study, it is found that $\sigma_{D C}$ shows a decreasing tendency as the temperature rises indicating a partial breakdown of the CB network in the host matrix. In addition, the $\mathrm{CB}$ network restricts the motions of the polymer chains above the glass transition temperature of the polymer and consequently increase the thermal stability as is probed by thermogravimetric analysis tests. Here, attempt has made to investigate the stability of the 
electrical properties under dynamic external filed as function of temperature in terms of the effective complex permittivity. Three selected composite samples EBA/CB were studied. The frequency of the applied field wastaken between 100and $10^{7} \mathrm{~Hz}$ for various temperatures in the range $10-70^{\circ} \mathrm{C}$.

\section{Experimental}

\subsection{Materials}

The samples of EBA copolymer filled with acetylene carbon black used in this investigation were obtained from Borealis AB (Sweden)[9]. The butylacrylate monomer (EBA) contains butylester side groups, providing a certain polarity and a relatively low crystallinity (about $20 \%$ in volume). The glass temperature transition of the neat polymer matrix is $T_{g}=-75^{\circ} \mathrm{C}[10]$. A three samples with nominal carbon black volume fraction has been studied.

\subsection{Differential thermal analyzes (ATD)}

The differential thermal analyzes (ATD) are carried out between temperatures of 20 to $180{ }^{\circ} \mathrm{C} .20 \mathrm{mg}$ of sample are placed in a platinum crucible. The acquisition takes place under ambient conditions and the heating rate is programmed at a rate of $10{ }^{\circ} \mathrm{C} / \mathrm{min}$.

\subsection{Raman measurements}

Raman spectra were collected at room temperature using Invia Reflex device (Renishaw). The laser line $514 \mathrm{~nm}$ was used. The scattered light was collected in backscattering configuration with a holographic grating (2400 lines $/ \mathrm{mm}$ ) to diffract the light on a CCD detector. The frequency range studied was $900-2000 \mathrm{~cm}^{-1}$

\subsection{Dielectric measurements}

For the dielectric measurements, the samples were prepared as square and the electrical contacts were formed by sputtering gold-palladium of $100 \mathrm{~nm}$ thickness on the opposite sites of the sample. The measurements were carried out, in the frequency $(F)$ range from 100to $10^{7} \mathrm{~Hz}$ and temperature from 10 to $70^{\circ} \mathrm{Cusing}$ an Agilent 4294A precision impedance analyzer. The permittivity may be a complex number quantity, in order to represent both stored energy and losses within the material. Under dynamic external fields, the permittivity $\varepsilon$ and conductivity $\sigma$ are related via equation:

$\varepsilon=\varepsilon^{\prime}-i \varepsilon^{\prime \prime}=\varepsilon^{\prime}-i\left(\varepsilon_{d}^{\prime \prime}+\frac{\sigma}{2 \pi F \varepsilon_{0}}\right)(1)$

The two terms forming the imaginary component represent polarization $\left(\varepsilon_{d}^{\prime \prime}\right)$ and conduction $\left(\frac{\sigma}{2 \pi F \varepsilon_{0}}\right)$ losses that it is not possible, in practice to be measured separately. $\varepsilon_{0}=8.854 \times 10^{-12} \mathrm{~F} \mathrm{~m}^{-1}$ is the permittivity of vacuum.

\section{Results and Discussion}

The differential thermal analysis (DTA) for elastomer matrix filled with different $\mathrm{CB}$ volume fractionreported in Figure 1.TheDTA curves show a small peak endothermic between $75^{\circ} \mathrm{C}$ and $98^{\circ} \mathrm{C}$. Melting peak indicates the melting of crystals part on the composite. The peaks have become broader and lower with increasing the filler volume fraction indicating the effect of $\mathrm{CB}$ on the thermal stability of the matrix. This result agree with the data obtained from the thermogravimetric analysis (TGA) [9].

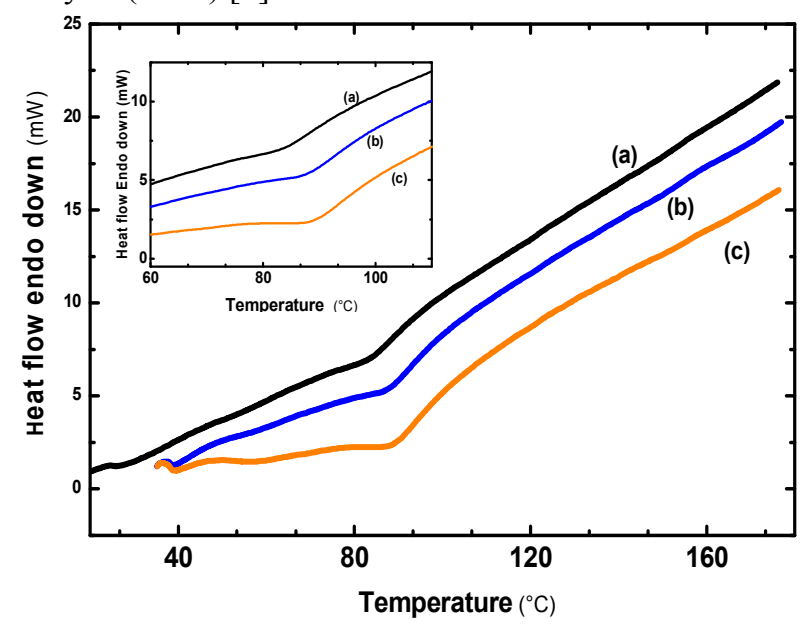

Fig. 1.DTA curves of ethylene-co-butyl acrylate polymer filled with three volume fractions of carbon black: (a) $6.8 \%$, (b) $15.8 \%$ and (c) $19.9 \%$ versus of temperature.

Raman Spectroscopy was used to assess the affinity between the $\mathrm{CB}$ particles and the EBA elastomeric matrix. The Raman spectra of the CB/EBA composite samples are reported in the Figure 2 with twotypical peaks located approximately at 1331 and $1580 \mathrm{~cm}^{-1}$ that are assigned to the disordered graphite structure (D band) and tangential stretching mode of carbon-carbon bands(G Band). The Raman spectra exhibit well defined Raman peaks for $\mathrm{CB}$ showing that their structural characteristics are not affected when the carbon black are embedded in the polymer matrix [11]. 


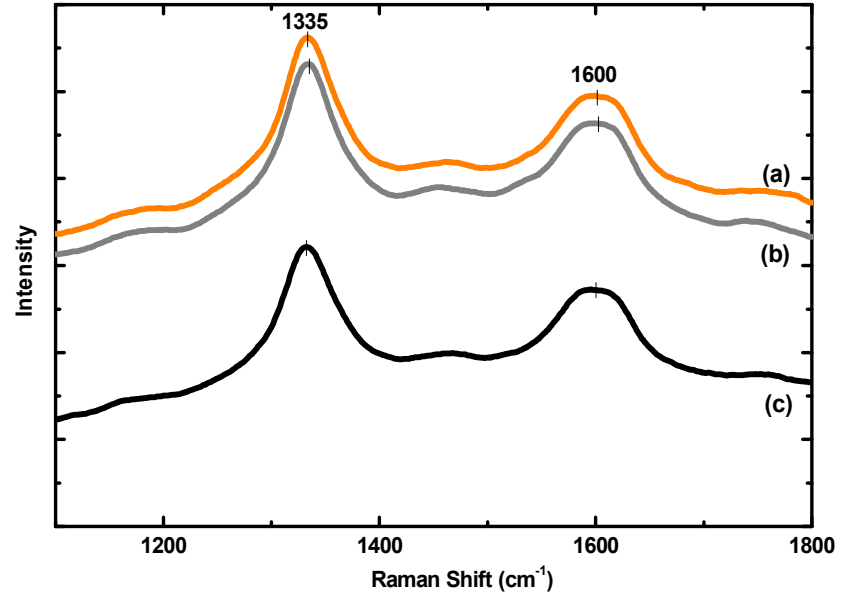

Fig. 2. Raman spectra of ethylene-co-butyl acrylate polymer filled with volume fractions of carbon black: (a) $6.8 \%$, (b) $15.8 \%$ and (c) $19.9 \%$

We turn now to the main focus of our study and examine the temperature dependence of the effective complex permittivity. The obtained spectra exhibit high values of both parts of the complex permittivity in the range of frequency considered here indicating a best dispersion of $\mathrm{CB}$ into the EBA matrix. In this frequency range, the spectra variation is generally attributed to the interfacial polarization [12] that occurs in heterophase materials. Figure 3 represents thevariation of real $\varepsilon^{\prime}$ and imaginary $\varepsilon^{\prime \prime}$ parts of the complex permittivity $\varepsilon$ versus the temperature for threeCB volume fractions at two frequencies (a) of $10 \mathrm{kHz}$ and (b) $1 \mathrm{MHz}$ representative of capacitor or actuator applications. Three remarks can be addressed. Firstly, both $\varepsilon^{\prime}$ and $\varepsilon^{\prime \prime}$ increase with increasing $\mathrm{CB}$ volume fractions. This trend is obvious sincethe density of $\mathrm{CB} / \mathrm{EBA}$ interfaces increases by adding more $\mathrm{CB}$ amount. In fact, theattractive interactions between fillers and polymer result in a socalled constrained layer[13]. The volume fraction of constrained polymer chain $V_{c}$ can be estimated according to the dynamic mechanical analysis (DMA)[13].The $V_{c}$ values that are $0.342,0.375$ and 0.422 are found to increasewith the three $\mathrm{CB}$ concentrations $6.8,15.8$ and $19.9 \%$.Secondly, we observea slight decrease of the imaginary part $\varepsilon^{\prime \prime}$ with temperature for all composites samples as can be seen in Figures 3. This behavior agree with the result reported in the Ref 9 concerning the dc electrical conductivity versus temperature and is attributed to the thermal expansion coefficient (TEC) of the matrix which is larger than that of the CB $[14,15]$.Thermal expansion resulting from temperature increase causes greater separation between the $\mathrm{CB}$ particles and hence leading to a decrease of losses.However, the real part $\varepsilon^{\prime}$ behaves differently with temperature. When the temperature exceeds $40^{\circ} \mathrm{C}$ a significant decrease is clearly observed especially for high loading samples. We suggest that TEC has more effecton the $\varepsilon^{\prime}$ than that on $\varepsilon^{\prime \prime}$ and consequently reduces the energy stored in the composite material.

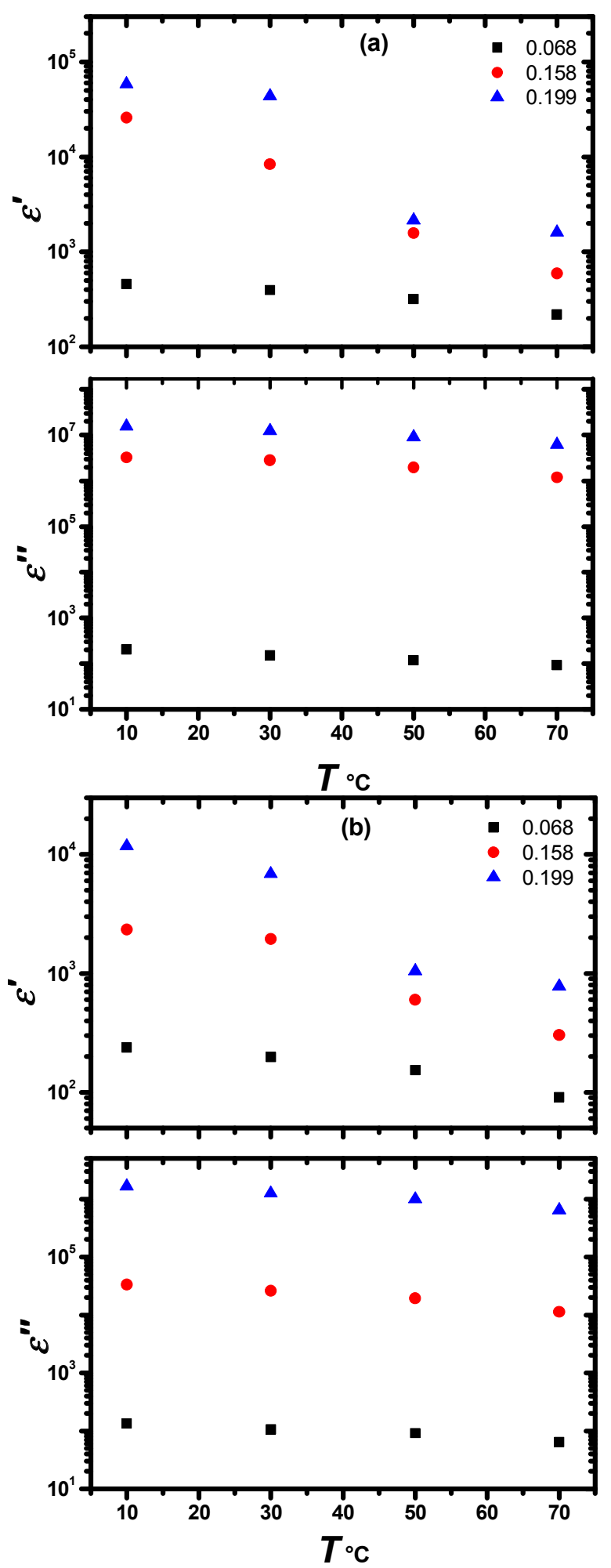

Fig. 3. Temperature dependence dependenceof real and imaginary parts of the effective complex permittivity of $\mathrm{EBA} / \mathrm{CB}$ for three volume fractions of $\mathrm{CB}$ at two frequencies (a) $10 \mathrm{kHz}$ and (b) $1 \mathrm{MHz}$

\section{Conclusion}


This paper probesthe dielectric propertiesof nanocomposites formed by incorporating highly conductive carbon black particles in an insulating ethylene butylacrylate matrix. The effective complex permittivity stability in function of temperature is studied in a radio frequency range and above the glass transition temperature $\left(T_{g}\right)$ of the polymer matrix. At the first time, both real $\varepsilon^{\prime}$ and imaginary $\varepsilon^{\prime \prime}$ parts of the complex permittivity are found to increase with the $\mathrm{CB}$ amount. The volume fraction of constrained polymer chain $V_{c}$ calculated from the DMA tests is used to explain the effect of the $\mathrm{CB}$ on dielectric response of the matrix. Secondly, the effect of the temperature on the complex permittivity exhibits a difference behavior between the

\section{References}

1. H. S. Nalwa, Handbook of Organic-Inorganic Hybrid Materials and Nanocomposites, (2003)

2. N. Lombardo,compos scitechnol67, 728-736 (2007)

3.T. A. Ezquerra, F. Kremer, and G. Wegner, in Dielectric Properties of Heterogeneous Materials, Progress in Electromagnetics Research, edited by A. Priou, ed. (Elsevier, New York, 1992)

4. C. Brosseau, J. Phys. D 39, 1277 (2006).

5.Mdarhri A, Brosseau C. Prospects in filled polymers engineering: mesostructure, elasticity network, and macroscopic properties. Brosseau $\mathrm{C}$, editor. Trivandrum: Research Signpost ( 2008)

6. V. Bobnar, B. Vodopivec, A. Levstik, M. Kosec, B. Hilezer, and Q .Zhang, Macromolecules, 36, 4436 (2003)

7. Y. Rao, S. Ogitani, P. kohl, and C. Wong, J. Appl. Polym. Sci. 83, 1084 (2002)

8. Bibhu Prasad Sahoo, KinsukNaskar, K. AbhinavDubey, Ram N. P. Choudhary, Deba Kumar Tripathy, , J Mater Sci48, 702-713 (2013)

9.A. Mdarhri, C. Brosseau, M. Zaghrioui, and I. El Aboudi, J. Appl. Phys. 112, 034118 (2012)

10.C. Brosseau, A. Mdarhri, and A. Vidal,J. Appl. Phys. 104, 074105/1-9 (2008)

11. Bokobza, J. Bruneel, M. Couzi, journal of carbon research 1, 77-94 (2015)

12. A.Patra , D. K.Bisoyi, J Mater Sci45, 57425748 (2010) 45:5742-5748

13. F. Elhaouzi, A. Nourdine, A. Mdarhri, I. El Aboudi, M. Zaghrioui,Polym Compos. (to be published) real and imaginary parts of the permittivity. A small decrease is only observed for the imaginary part $\varepsilon^{\prime \prime}$ and it is attributed to the difference of the thermal expansion coefficient (TEC) of the matrix and CB particles. However, the real part $\varepsilon^{\prime}$ of the complex permittivity is found to be more sensitive to temperature especially for high loading composite samples. More investigations are needed for a best understanding of the permittivity stability in particularly at wide temperature and high frequency ranges.

\section{Acknowledgments}

The authors and especially F. Elhaouzi thank CNRSTMorocco for the Excellence Scholarship.

14. A. Sadezky, H. Muckenhuber, H. Grothe, R. Niessner, U. Poschl, Carbon 43, 1731-1742 (2005)

15. R.N. Rothon, Particulate-Filled Polymer Composites, 2nd Edition, (United Kingdom 2003) 